\title{
CALIDAD DE VIDA EN PACIENTES RENALES HEMODIALIZADOS
}

\author{
QUALITY OF LIFE ON PATIENTSHAEMODIALYSIS
}

\author{
MAGALI RODRÍGUEZ VIDAL*, MANUEL CASTRO SALAS** \\ Y JOSÉ M. MERINO ESCOBAR***
}

\begin{abstract}
RESUMEN
Estudio descriptivo de corte transversal, cuyo propósito fue conocer las características personales y calidad de vida de pacientes con insuficiencia renal crónica en tratamiento sustitutivo con hemodiálisis. El grupo de estudio estuvo constituido por 90 pacientes de dos centros de diálisis de la ciudad de Concepción, Chile. La calidad de vida se midió a través del cuestionario genérico de salud SF-36. Aproximadamente la mitad de los pacientes tenían entre 45 y 64 años, la distribución por sexo fue prácticamente similar, tres cuartas partes de los sujetos se declararon con pareja , más de la mitad poseía baja escolaridad y todos poseían algún sistema previsional. El valor más alto de calidad devida fue 81,8 y el más bajo 3,25. Por lo que se pudo concluir que aproximadamente la mitad de las personas encuestadas tenía una calidad de vida por sobre el nivel promedio.
\end{abstract}

Palabras claves: Calidad de vida, insuficiencia renal, hemodiálisis.

\begin{abstract}
ABST RACT
Cross sectional descriptive study whose purpose was to know personal characteristics and quality of life of chronic renal failure patients on haemodialysis as substitutivetherapy. N inety patients from two dialysis centres located at Concepción, Chile, were the study group. SF-36 generic health questionnaire was used to measure quality of life. Almost half of patients were 45 to 65 years old, had an equivalent gender distribution, three fourths of subjects declared coupled, more than half had low schooling and all of them had a health insurance system. Results showed that the highest value for quality of life was 81.8 and the lowest, 3,25 . We conclude that almost half of inquiered subjects had a quality of life above average level.
\end{abstract}

Keywords: Quality of life, Renal failure, H aemodialysis.

Recepción: 20/06/05. Aceptación: 07/11/05

\section{INTRODUCCIÓN}

El interés por estudiar la calidad de vida ( $C$. de V.) es antiguo, sin embargo la preocupación por el desarrollo sistemático y científico del constructo es relativamente reciente, la idea comienza a popularizarse en la década del sesenta, hasta convertirse hoy en un concepto utilizado en ámbitos muy diversos, como la salud, la educación, la economía, la política y el mundo de los servicios en general (Gómez, 2000).

H asta los años 60, el creciente interés por conocer el bienestar humano y la preocupación por las consecuencias de la industrialización experimentada por la sociedad, hacen surgir la necesidad de medir esta realidad a través de datos objetivos (relacionados con

\footnotetext{
* Profesor Asistente, Magister en Enfermería Universidad Concepción, Concepción-Chile. Dirección: Roosevelt s/n, telefonos: (56-41) 204826 / (56-41) 2070 64, fax: (56-41) 228353. E-mail: magrodri@udec. cl

** Profesor Asociado, Magister en Enfermeria, Sociólogo, Universidad de Concepción, Concepción-Chile. Dirección: Roosevelt s/n telefonos: (56-41) 204826 - (56-41) 207064, fax: (56-41) 228353. E-mail: mcastro@udec. cl

*** Profesor Titular. Sociólogo, M agíster en Ciencias Sociales, PhD in Sociology, Universidad de Concepción, ConcepciónChile. Dirección: Roosevelt s/n, telefonos: (56-41) 204826 - (56-41) 2070 64, fax: (56-41) 228353. E-mail: jmerino@udec. cl
} 
las necesidades del individuo en salud, educación, etc.) y en términos subjetivos mediante el análisis de la percepción del individuo en cuanto a lo que considera su nivel debienestar.

La diferencia entre los indicadores objetivosy subjetivos determinarían los componentes de la misma del individuo (Palomino y López, 1999). Existeconsenso entrelos diversos autores que han abordado el tema, que la C. de V. está relacionada con las necesidades humanas, y que la evaluación de ésta debe hacerse a través de datos objetivos y subjetivos (M oreno, 1996).

En los años 80 el término calidad de vida se adoptó como un concepto sensibilizador que podía ofrecer a los profesionales de distintas disciplinas un lenguaje común y guiar las prácticas de los servicios humanos, más orientados hacia la persona, su autodeterminación y el logro de una mayor satisfacción con su vida (Jiménez, 1998).

A lo largo de los 90 las preocupaciones en torno ala definición y evaluación del concepto tuvieron un mayor carácter metodológico. Superadas estas inquietudes, el siglo XXI se presenta como aquél en que el término calidad de vida no sólo teñirá las intenciones y acciones de individuos que gozan cada vez de mayores posibilidades de elección y decisión y optan por una vida de mayor calidad, sino también la delos servicios humanos en general, que se verán obligados a adoptar técnicas de mejoramiento de sus procedimientos, en la medida que existirá un grupo de evaluadores que analizará sus resultados desde criterios deexcelencia como es el de calidad devida (Croog y Levine, 1989).

Numerosos trabajos deinvestigación científica emplean hoy el concepto, como un modo de referirse a la percepción que tieneel paciente de los efectos de una enfermedad determinada, especialmentedelas consecuencias que provoca sobre su bienestar físico, emocional y social (Lugones, 2002). En las ciencias de la salud los avances de la medicinahan hecho posibleprolongar notablemente la vida, generando un aumento importante de las personas con enfermedades crónicas, lo que ha llevado a poner especial acento en la Calidad de Vida Relacionada con Salud (CVRS) (Alvares y Badía, 1995).

La CVRS ha probado ser útil, principalmente como un rasgo de estructura general, dentro de la cual las dimensiones relevantes de la vida y salud se pueden examinar en investigación y prácticas clínicas (Dubos, citado por Croogy Levine, 1989). Su medición se ha utilizado para distinguir diferencias entre pacientes 0 grupos de pacientes, para predecir resultados en ellos y para evaluar las intervencionesterapéuticas (Bravo y Falache 1993).

A partir dela década de los 80 , el concepto fue aplicado en el mundo de las enfermedades incapacitantes, dado que captaba una visión nueva y cambiantesobrelas personas con discapacidad (Schwartzmann, 2003). En la medida quela satisfacción con la vida se consideró muy ligada a las posibilidades de tomar decisiones y elegir entre diversas opciones, se abrieron oportunidades a las personas con discapacidad para expresar sus gustos, deseos, metas y aspiraciones y a tener mayor participación en las decisiones que las afectan (Gómez y Sabeh citando a Schalok, 2000).

Los avances tecnológicos experimentados en las últimas décadas han llevado a un considerableaumento deherramientas biotecnológicas en salud, en contraste con el lento de sarrollo deherramientas vinculadas a los factores 0 aspectos sociales y humanos involucrados (Velardey Ávila, 2002). Esto lleva a que se produzca un desequilibrio en la atención de salud, con un acentuado énfasis en el diagnóstico y tratamiento de las enfermedades y una insuficiente consideración de las consecuencias psicosociales de sus problemas ( $R e$ bollo y col., 2000).

La aplicación de la tecnología previene la muerte prematura, como ocurre con las enfermedades crónicas terminales, pero a su vez sobreviene una serie de problemas que se re fieren a las consecuencias de vivir con ellas. Las enfermedades cardíacas y renales reflejan 
plenamente los problemas de costo y efectos sobre la C. de V. de los pacientes portadores de estas patologías ( Lawrence y Gaus, citado por Croog y Levine, 1989).

La rehabilitación de los pacientes con enfermedad renal en diálisis es un objetivo fundamental delos programas detratamiento de la Insuficiencia Renal Crónica Terminal (IRCT) (Iborra y col., 1998). Los parámetros fisiológicosy bioquímicos suministran información muy valiosa, pero lo que realmente interesa a los pacientes es la manera como la enfermedad y los tratamientos que reciben afectan su capacidad funcional y su C. de V. en general (Zanoguera y col., 1998).

Para enfermería profesional el estudio de la C. deV. del individuo es de gran interés, ya que le permite conocer al ser humano desde una perspectiva más integral, que incluye sus valores, creencias y percepciones. Este conocimiento le permitiría realizar intervenciones de acuerdo a las características y situación de vida por la que está atravesando. El quehacer de enfermería debería centrarse en ayudar a losindividuos a satisfacer más plenamentesus necesidades, incrementando su conocimiento de las múltiples alternativas que tienen en relación con su salud y así determinar las orientaciones devalor para cada uno de ellos.

El número de pacientes con IRCT tratados mediante hemodiálisis (H.D.) ha experimentado un significativo aumento en Chile, reflejando lastendencias denaciones más desarrolladas. Así, la tasa de pacientes tratados ha cambiado, desde 12,7 personas por millón de habitantes en 1980, a 18,3 personas por millón de habitantes en 1992, haciendo la $H$.D. accesible a pacientes que no son candidatos a transplante renal y que tienen en ésta la única y definitiva forma de tratamiento (Poblete, 1999). Aunque no cabe duda que la $H$.D. es efectiva para prolongar la vida del paciente con nefrópatía, existe una controversia considerable respecto a la calidad de esta sobrevida, más aún cuando nuevas modalidades dediálisissehan centrado en los potenciales beneficios queellas ofrecen para mejorar la
C. deV. de los pacientes (Ureña y col., 1997).

Esinteresante, entonces, para nuestra profesión saber ¿cuál es la calidad de vida y las características personales de los pacientes sometidos a hemodiálisis? La respuesta a esta interrogante nos permitiría hacer un aporte y prepararse para la atención de estos pacientes y otros con enfermedades crónicas, que están experimentando un incremento acelerado en nuestro país.

Por lo tanto, el propósito de este estudio fue conocer la calidad de vida y las características personales de los pacientes con insuficiencia renal crónica en tratamiento sustitutivo con hemodiálisis.

\section{METODOLOGÍA}

\section{Estudio cuantitativo, descriptivo de corte transversal}

El universo deesta investigación estuvo constituido por 305 personas, que es el total de personas que estaban en tratamiento con he modiálisisen laVIII Región Chile(INE, 2001). La muestra de 110 pacientes hemodializados fue tomada desde el Centro Regional de Diálisis Limitada (70 pacientes) y del Centro Regional Tal cahuano Limitada (40 pacientes), ya que entre ambos atienden a un 36\% del total de los pacientes de la región. Se daban garantías de tratamiento adecuado, contando con tecnología de última generación y atención a pacientes subvencionados en su mayoríapor el Fondo Nacional deSalud (FONASA). La muestra incluyó pacientes en tratamiento durantelos meses deabril, mayo y junio 2001.

Previo a la aplicación del instrumento, los pacientes recibieron una copia del consentimiento informado, el cual les fueleído por el encuestador. Se aclararon las dudas que surgieron y luego el pacientefirmó la aceptación para ser incluido en esta investigación.

Se consideraron los siguientes criterios de exclusión: 
- Meses en tratamiento sustitutivo con HD: Pacientes que registraran menos de tres meses en tratamiento con H.D. y que por las características del tratamiento dialítico no se encontraban estabilizados (7 pacientes).

- Condiciones psíquicas inadecuadas: Seconsideró sólo el deterioro intelectual severo como motivo de exclusión (5 pacientes), medido a través del $M$ inimental Test.

- Ceguera: Ya que la C. deV. de estos pacientes se encontraba deteriorada, por la existencia de una patología previa (8 pacientes).

La muestra quedó constituida finalmente por 90 pacientes adultos, entre 22 y 89 años de edad, portadores de Insuficiencia Renal Crónica Terminal en tratamiento sustitutivo con Hemodiálisis, que desde abril del 2001 tenían a lo menostresmesesen tratamiento dialítico.

Para medir la variable principal "Calidad de Vida" se utilizó el Cuestionario de Salud SF-36 que fue desarrollado por Ware (1993), adaptado y validado en España por Alonso y col. (1995), para medir conceptos genéricos de salud relevantes a través de la edad, enfermedad y grupos detratamiento. Proporciona un método exhaustivo, eficientey psicométricamente sólido para medir la salud desde el punto de vista del paciente, puntuando respuestas estandarizadas a preguntas estandarizadas. El cuestionario final cubre 8 dimensionesdel estado desalud; contiene 36 ítemesque cubren dosáreas, el estado funcional y el bienestar emocional (Schwartzmann y col., 1999).

Las 8 dimensiones medidas son:

- Función Física (FF): grado en que la salud, limita las actividades físicas tales como el autocuidado, caminar, inclinarse, realizar esfuerzos etc. (10 pts).

- Rol Físico(RF): grado en que la salud física interfiere en el trabajo y en otras actividades diarias incluyendo rendimiento menor que el deseado. (4 pts).

- Dolor Corporal (DC): la intensidad del dolor y su efecto. (10 pts).

- Salud General (SG): valoración personal de la salud que incluyela salud actual, las perspectivas de salud en el futuro y la resistencia a enfermar. (20 pts).

- Vitalidad (V): sentimiento de energía y vitalidad, frenteal sentimiento de cansancio y agotamiento. (20 pts).

- Función Social (FS): grado en que los problemas de salud física o emocional interfieren en la vida social. (8 pts).

- Rol Emocional (RE): grado en que los problemas emocionales interfieren en el trabajo u otras actividades diarias, incluyendo reducción en el tiempo dedicado a éstas, rendimiento menor y disminución del esmero en el trabajo. (3 pts).

- Salud Mental (SM ): salud mental general, incluyendo depresión, ansiedad, control de la conducta y el bienestar. ( 25 pts).

Total del puntaje: 100 pts.

Las variables independientes de este estudio fueron medidas a través de un cuestionario constituido por 15 preguntas que fue elaborado por la autora.

La recopilación de datos se hizo mediante una entrevista en que se aplicó el instrumento como un todo. La entrevista fue realizada por la investigadora a cada paciente en la antesala de la unidad de hemodiálisis, esperando la entrada o salida de la unidad o durante el tratamiento dialítico. El tiempo empleado dependió de las características personales del encuestado, variando entre treinta a cuarenta minutos como máximo.

En la primera parte de la entrevista se obtuvo la información relativa a los criterios de exclusión, con el fin de retirar a los pacientes queno cumplían con los requisitosparaintegrar la muestra, y luego se procedió a aplicar el cuestionario que permitió dar respuesta a los objetivos de esta investigación.

El control de calidad de los datos para el Cuestionario de Salud SF-36 se realizó a través del Alfa de Cronbach, dando como resultado para cada una de las escalas lo siguiente:

Función Física: 0,93; Rol Físico: 0,83; DoIor Corporal: 0,92; Salud General: 0,73; Vita- 
lidad: 0,84; Rol Social: 0,83; Rol Emocional: 0,84; Salud M ental: 0,80.

Como podemos observar todos los valoresestán sobre 0,7, lo quenosindica una gran confiabilidad del instrumento SF-36.

Lainformación recolectada fuecodificada y procesada computacional mentemedianteel programa estadístico SAS, en la División de Computación e Informática de la Universidad de Concepción.

Para el examen de la información obtenida en esta investigación se ha utilizado como estrategia deanálisis el model o general lineal, que implica una variable dependiente continua. Se ha utilizado este modelo, pues se parte del supuesto quelas variables se relacionan linealmente. Se piensa aquí que un modelo lineal tiene sentido y funciona bien en la práctica investigativa que nos preocupa.

Se ha considerado la variable dependiente (Calidad deVida) como continua, porquetieneuna escalatrasformada queincluyeun rango de 0 a 100 puntos.

\section{RESULTADOS}

Las características personales de la población estudiada se presentan a continuación:

Los pacientes estudiados exhiben edades que oscilan entre 22 y 89 años de edad, el mayor número depacientes $45,6 \%$ seencuentra entre la población adulto maduro (45 y 64 años) y adulto mayor con un 36,6\% (65 años y más), siendo minoritario el grupo de adultos jóvenes con un 17,8 (22 y 44 años).

Considerando el sexo, la proporción es muy similar, hombres $48,9 \%$ y mujeres $51,1 \%$.

En cuanto a la situación marital, $65,6 \%$ se declara con pareja, y un 34\% sin pareja. Respecto al nivel de instrucción un $55,6 \%$ posee una baja escolaridad, es decir analfabeto, educación básica completa eincompleta. Del grupo en estudio un $88,9 \%$ declaró practicar alguna religión. Un $62,2 \%$ refiere tener actividad laboral, y un $100 \%$ de los pacientes tiene previsión.
En relación a las principales causas de ingreso, un $43,3 \%$ corresponde a nefropatía diabética, $34,4 \%$ a causas desconocidas y un $22 \%$ a otrasquecorresponden a glomerulonefritis crónica, uropatías obstructivas y lupus eritematoso.

Losmeses en tratamiento dialítico fluctuaron entre 4 y 168 meses.

Se pudo constatar que la distribución de la variablecalidad devida es simétrica, ya que la mediana $(44,6)$ es similar al promedio $(44,8)$, los cuartiles tienen distancias similares y el gráfico de probabilidad normal nos dicequela mitad de las personas encuestadas aproximadamentetienen una calidad de vida por sobre el nivel del promedio. En una escala transformada de 0 a 100 , el puntaje encontrado con mayor calidad devidafuede81,8, mientras que el valor más bajo fue de 3,25 puntos.

Dado que la variable dependiente se aproxima bastanteal modelo dela curva normal, se pueden aplicar los cálculos de medidas de los modelos generales lineales, como la T de Student, AN OVA, y Regresión lineal con absoluta confianza.

Examinando cada uno de las dimensiones quecomponen la escala se pudo observar que la calidad de vida en esta población es regular a baja, ya que las medias aritméticas fueron las siguientes: Función Física $(64,7)$, Rol Social $(55,9)$ y Rol Emocional $(52,5)$ obtuvieron las puntuaciones más altas, y las dimensiones Rol Físico $(23,6)$, Vitalidad $(38,2)$ y Salud M ental $(38,1)$ obtuvieron las puntuaciones más bajas.

El examen decada una de las dimensiones del cuestionario de salud SF-36 indica que:

- Función física: Un 76,7\% de los pacientes se encuentra con algún grado de limitación, para realizar esfuerzos intensos, tales como correr, levantar objetos pesados y realizar deportes agotadores.

En relación a los esfuerzos moderados, un $61,1 \%$ se encuentra limitado en algún grado, para caminar más de una hora, mover una mesa, etc. 
El 63,3\% presenta dificultad para subir varios pisos por la escalera, y un 44\% presenta dificultad para agacharse 0 arrodillarse.

- Un 56,7\% tiene algún grado de limitación para caminar un kilómetro, pero un $65,6 \%$ refiere no presentar dificultad para caminar una o varias manzanas.

- La gran mayoría 88,9\% de los pacientes con insuficiencia renal crónica terminal, refiere que puede vestirse y/o bañarse por sí mismo y sólo un $6,7 \%$ se encuentra muy limitado para hacerlo (Tabla № I).

- Rol Físico: En todoslositemes, sobre un 70\% de los pacientes declara que su salud física interfiere en el desempeño de su trabajo y en la realización de sus actividades cotidia- nas, incluyendo rendimiento menor que el deseado (Tabla $\mathrm{N}$ ㅇII).

- Rol Emocional: Entre 44,4 y 51,1\% de los pacientes reconoce la existencia de problemas emocionales en las actividades cotidianas y/o laborales (Tabla $\mathrm{N}$ ㅇII).

- Rol Social: Delas respuestas se aprecia quea un $64,4 \%$ de los pacientes (al sumar algunas veces, sólo al guna vez y nunca) durante las últimas 4 semanas, la salud física o los problemas emocionales no le han dificultado realizar sus actividades sociales como visitar amigos, vecinos o parientes, y que un $35,6 \%$ sí ha tenido dificultad para desarroIlar sus actividades sociales habituales ( Tabla $\mathrm{N} \cong \mathrm{II}$ ).

Tabla N 이. Función física de pacientes con Insuficiencia Renal Crónica Terminal sometidos a tratamiento con hemodiálisis durante los meses de abril, mayo y junio 2001. Chile.

\begin{tabular}{|l|c|r|r|r|r|r|}
\hline \multirow{2}{*}{ Función física } & \multicolumn{2}{|c|}{$\begin{array}{c}\text { Sí melimita } \\
\text { mucho }\end{array}$} & \multicolumn{2}{c|}{$\begin{array}{c}\text { Sí melimita } \\
\text { un poco }\end{array}$} & \multicolumn{2}{c|}{$\begin{array}{c}\text { No me limita } \\
\text { nada }\end{array}$} \\
\cline { 2 - 8 } & $\mathbf{N}$ o & $\%$ & No & $\%$ & No & $\%$ \\
\hline $\begin{array}{l}\text { Esfuerzos intensos tales como correr, } \\
\text { levantar objetos pesados, practicar } \\
\text { deportes agotadores }\end{array}$ & 48 & 53,4 & 21 & 23,3 & 21 & 23,3 \\
\hline $\begin{array}{l}\text { Esfuerzos moderados como mover una } \\
\text { mesa, caminar más de una hora }\end{array}$ & 28 & 31,1 & 27 & 30 & 35 & 38,9 \\
\hline Llevar o levantar la bolsa de compras & 21 & 23 & 24 & 23,1 & 44 & 50,9 \\
\hline Subir varios pisos por la escalera & 23 & 25,6 & 34 & 37,7 & 33 & 36,7 \\
\hline Subir un sólo piso por la escalera & 13 & 14,4 & 16 & 17,8 & 61 & 67,8 \\
\hline Agacharse o arrodillarse & 18 & 20 & 22 & 24 & 50 & 56,6 \\
\hline Caminar un kilómetro o más & 35 & 38,9 & 16 & 17,8 & 39 & 43,3 \\
\hline Caminar una manzana & 13 & 14,4 & 18 & 20 & 59 & 65,6 \\
\hline Bañarse o vestirse por sí mismo & 6 & 6,7 & 4 & 4,4 & 80 & 88,9 \\
\hline Caminar varias manzanas & 13 & 14,4 & 18 & 20 & 59 & 65,6 \\
\hline
\end{tabular}

Fuente: Calidad de vida en pacientes renales hemodializados. 
Tabla No II. Rol físico y emocional de pacientes con Insuficiencia Renal Crónica Terminal sometidos a tratamiento con hemodiálisis durante los meses de abril, mayo y junio 2001. Chile.

\begin{tabular}{|c|c|c|c|c|}
\hline \multirow{2}{*}{$\begin{array}{l}\text { Rol físico } \\
\text { Durante las últimas } 4 \text { semanas ¿ha tenido alguno de los si- } \\
\text { guientes problemas en su trabajo o actividades }\end{array}$} & \multicolumn{2}{|c|}{ Sí } & \multicolumn{2}{|c|}{ No } \\
\hline & No & $\%$ & № & $\%$ \\
\hline $\begin{array}{l}\text { Tuvo que aumentar el tiempo dedicado al trabajo o a sus } \\
\text { actividades cotidianas }\end{array}$ & 63 & 70 & 27 & 30 \\
\hline Hizo menos de lo que hubiera querido hacer & 73 & 81,1 & 17 & 18,9 \\
\hline $\begin{array}{l}\text { Tuvo que dejar de hacer al gunas tareas en su trabajo o en } \\
\text { sus actividades cotidianas }\end{array}$ & 73 & 81,1 & 17 & 18,9 \\
\hline $\begin{array}{l}\text { Tuvo dificultad para hacer su trabajo o susactividades coti- } \\
\text { dianas (por ejemplo le costó más de lo normal) }\end{array}$ & 66 & 73,3 & 24 & 26,7 \\
\hline Rol emocional & & & & \\
\hline $\begin{array}{l}\text { ¿Tuvo que aumentar el tiempo dedicado a su trabajo o a sus } \\
\text { actividades cotidianas por algún problema emocional? }\end{array}$ & 40 & 44,4 & 50 & 55,6 \\
\hline $\begin{array}{l}\text { ¿H izo menos de lo que hubiera querido hacer por al gún } \\
\text { problema emocional? }\end{array}$ & 46 & 51,1 & 44 & 48,9 \\
\hline $\begin{array}{l}\text { ¿No hizo su trabajo o sus actividades cotidianas tan cuida- } \\
\text { dosamente, por algún problema emocional? }\end{array}$ & 42 & 46,7 & 48 & 56,3 \\
\hline
\end{tabular}

- Dolor Corporal: Con respecto a esta dimensión al sumar las categorías se aprecia que $64,4 \%$ de los pacientes presenta dolor en alguna parte de su cuerpo.

Durante las últimas cuatro semanas un $45,6 \%$ afirma haber presentado muy poco o nada de dolor, mientras que aquellos que informan haber sido comprometidos mayormente por estesíntoma, 54\% refiereque el dolor en algún grado les ha dificultado realizar su trabajo habitual (Tabla $\mathrm{N} N$ III).

- Energía y Vitalidad: A proximadamente el $50 \%$ de los pacientes establece que siempre o casi siempre se siente lleno de vitalidad y/ o energía, mientras la otra mitad declara sentirse agotado o cansado casi todo el tiempo (Tabla No IV).

- Salud M ental: Con respecto a esta dimensión, seobservó queun $71,1 \%$ delos pacientes manifiesta que sólo algunas veces, alguna vez o nunca ha estado nervioso, mien- tras que un 28,9\% reconoce tener esa condición siempre o casi siempre. Un $44,5 \%$ de ellos presentó en algún grado sentimientos de tristeza y desánimo.

En relación a sentimientos de felicidad, un 37,8\% manifestó estar siempre o casi siemprefeliz, mientras que un $62,3 \%$ lo está sólo ocasionalmente (Tabla № IV).

- Salud General: En cuanto a esta dimensión, un $65,6 \%$ percibe que su salud es regular a mala, un tercio $(31,1 \%)$ la cataloga de buena y sólo un 3,3\% manifiesta una excelente y muy buena salud.

Dos tercios de los entrevistados percibe no estar tan sano como cualquiera, y un $44,5 \%$ cree que su salud va a empeorar, un $28,9 \%$ no lo sabe, y un $26,6 \%$ cree que su salud va a mejorar con el tiempo. Un $70 \%$ de los encuestados no cree que su salud es excelente y sólo un $21,1 \%$ sí declara gozar de excelente salud (Tabla № V ) . 
Tabla N N III . D olor corporal y rol social de pacientes con Insuficiencia Renal Crónica Terminal sometidos a tratamiento con hemodiálisis durante los meses de abril, mayo y junio 2001. Chile.

\begin{tabular}{|c|c|c|c|c|c|c|c|c|c|c|}
\hline \multirow[t]{2}{*}{ Dolor corporal } & \multicolumn{2}{|c|}{ Nada } & \multicolumn{2}{|c|}{ Un poco } & \multicolumn{2}{|c|}{ Regular } & \multicolumn{2}{|c|}{ Bastante } & \multicolumn{2}{|c|}{ Mucho } \\
\hline & $\mathrm{N} \underline{0}$ & $\%$ & № & $\%$ & No & $\%$ & $\mathrm{~N} N$ & $\%$ & № & $\%$ \\
\hline $\begin{array}{l}\text { Tuvo dolor en alguna parte del cuerpo } \\
\text { durantelas últimas } 4 \text { semanas }\end{array}$ & 23 & 25,6 & 9 & 10 & 26 & 28,9 & 31 & 34,4 & 1 & 1,1 \\
\hline $\begin{array}{l}\text { Duranteslasúltimas } 4 \text { semanas hastaqué } \\
\text { punto el dolor le ha dificultado su tra- } \\
\text { bajo habitual }\end{array}$ & 26 & 28,9 & 15 & 16,7 & 12 & 13,3 & 33 & 36,7 & 4 & 4,4 \\
\hline Rol social & & & & & & & & & & \\
\hline $\begin{array}{l}\text { Durantelas últimas } 4 \text { semanas hasta qué } \\
\text { punto su salud física o los problemas } \\
\text { emocionales han dificultado sus activi- } \\
\text { dades sociales habituales, con la familia, } \\
\text { amigos, vecinos u otras personas. }\end{array}$ & 24 & 26,7 & 22 & 24,4 & 12 & 13,3 & 26 & 28,9 & 6 & 6,7 \\
\hline $\begin{array}{l}\text { Durante las últimas } 4 \text { semanas, con qué } \\
\text { frecuencia la S.F. o los problemas emo- } \\
\text { cionales le han dificultado sus activida- } \\
\text { des sociales. }\end{array}$ & 9 & 10 & 31 & 34,4 & 18 & 20 & 26 & 28,7 & 6 & 6,7 \\
\hline
\end{tabular}

Tabla No IV. Energía/Vitalidad y Salud M ental de pacientes con Insuficiencia Renal Crónica Terminal sometidos a tratamiento con hemodiálisis durante los meses de abril, mayo y junio 2001. Chile.

\begin{tabular}{|c|c|c|c|c|c|c|c|c|c|c|}
\hline \multirow[t]{2}{*}{ Energía y vitalidad } & \multicolumn{2}{|c|}{ Siempre } & \multicolumn{2}{|c|}{$\begin{array}{l}\text { Casi } \\
\text { siempre }\end{array}$} & \multicolumn{2}{|c|}{$\begin{array}{l}\text { Algunas } \\
\text { veces }\end{array}$} & \multicolumn{2}{|c|}{$\begin{array}{l}\text { Sólo } \\
\text { alguna vez }\end{array}$} & \multicolumn{2}{|c|}{ Nunca } \\
\hline & $\mathrm{N} \cong$ & $\%$ & № & $\%$ & № & $\%$ & $\mathrm{~N} N \underline{0}$ & $\%$ & № & $\%$ \\
\hline Se sintió lleno de vitalidad & 12 & 13,3 & 24 & 26,7 & 34 & 37,8 & 13 & 14,4 & 7 & 7,8 \\
\hline Tuvo mucha energía & 11 & 12,2 & 23 & 25,6 & 3 & 36,7 & 17 & 18,9 & 6 & 6,7 \\
\hline Se sintió agotado & 6 & 6,7 & 34 & 37,8 & 41 & 45,6 & 6 & 6,7 & 3 & 3,3 \\
\hline Se sintió cansado & 8 & 8,9 & 32 & 35,6 & 40 & 44,4 & 7 & 7,8 & 3 & 3,3 \\
\hline $\begin{array}{l}\text { Salud mental } \\
\text { ¿Estuvo muy nervioso? }\end{array}$ & 3 & 3,3 & 23 & 25,6 & 47 & 52,2 & 10 & 11,1 & 7 & 7,8 \\
\hline $\begin{array}{l}\text { ¿Se ha sentido tan deprimido } \\
\text { que nada le sube el ánimo? }\end{array}$ & 6 & 6,7 & 28 & 31,1 & 44 & 48,9 & 8 & 8,9 & 4 & 4,4 \\
\hline ¿Se sintió cal mado y tranquilo? & 8 & 8,9 & 26 & 28,9 & 36 & 40 & 19 & 21,1 & 1 & 1,1 \\
\hline ¿Se sintió desanimado y triste? & 8 & 8,9 & 32 & 35,6 & 41 & 45,5 & 6 & 6,7 & 3 & 3,3 \\
\hline ¿Se sintió feliz? & 6 & 6,7 & 28 & 31,1 & 32 & 35,6 & 14 & 15,6 & 10 & 11,1 \\
\hline
\end{tabular}


Tabla No V. Salud general de pacientes con Insuficiencia Renal Crónica Terminal sometidos a tratamiento con hemodiálisis durante los meses de abril, mayo y junio 2001. Chile.

\begin{tabular}{|c|c|c|c|c|c|c|c|c|c|c|}
\hline \multirow[t]{2}{*}{$\begin{array}{l}\text { Salud general } \\
\text { Item I }\end{array}$} & \multicolumn{2}{|c|}{ Excelente } & \multicolumn{2}{|c|}{$\begin{array}{l}\text { Muy } \\
\text { buena }\end{array}$} & \multicolumn{2}{|c|}{ Buena } & \multicolumn{2}{|c|}{ Regular } & \multicolumn{2}{|c|}{ Mala } \\
\hline & $\mathrm{N} N$ & $\%$ & $\mathrm{~N} \underline{0}$ & $\%$ & No & $\%$ & No & $\%$ & No & $\%$ \\
\hline En general ud. diría que su salud es: & 1 & 1,1 & 2 & 2,2 & 28 & 31,1 & 45 & 50 & 14 & 15,6 \\
\hline \multirow[t]{2}{*}{$\begin{array}{l}\text { Salud general } \\
\text { Item II }\end{array}$} & \multicolumn{2}{|c|}{$\begin{array}{l}\text { Todo } \\
\text { verdadero }\end{array}$} & \multicolumn{2}{|c|}{$\begin{array}{c}\text { Bastante } \\
\text { cierto }\end{array}$} & \multicolumn{2}{|c|}{ No lo sé } & \multicolumn{2}{|c|}{$\begin{array}{l}\text { Bastante } \\
\text { falsa }\end{array}$} & \multicolumn{2}{|c|}{$\begin{array}{l}\text { Totalmente } \\
\text { falsa }\end{array}$} \\
\hline & № & $\%$ & № & $\%$ & № & $\%$ & № & $\%$ & № & $\%$ \\
\hline $\begin{array}{l}\text { Creo que me pongo enfermo más } \\
\text { fácilmente que otras personas }\end{array}$ & 3 & 3,3 & 33 & 36,7 & 15 & 16,7 & 35 & 38,9 & 4 & 4,4 \\
\hline Estoy tan sano como cualquiera & 2 & 2,2 & 18 & 20 & 10 & 11,1 & 57 & 63,3 & 3 & 3,3 \\
\hline Creo que mi salud va a empeorar & 5 & 5,6 & 35 & 38,9 & 26 & 28,9 & 22 & 24,4 & 2 & 2,2 \\
\hline Mi salud es excelente & 1 & 1,1 & 18 & 20 & 8 & 8,9 & 52 & 57,8 & 11 & 12,2 \\
\hline
\end{tabular}

\section{DISCUSIÓN}

Analizando la variable dependientecalidad de vida, medida a través del cuestionario SF-36, y las dimensiones que la componen, se pudo observar que el $50 \%$ de las personas encuestadas tiene una calidad de vida por sobre el promedio. Al comparar las 8 dimensionesque componen la escala, se pudo observar que la función física, el rol emocional y el rol social obtuvieron las puntuaciones másaltas, mientras que el rol físico, vitalidad y energía, y salud mental, las puntuaciones más bajas.

En este estudio se encontró que la categoría función física alcanzó una media de $64,7 \%$ (DT:30) y en el análisis de sus ítemes se detectó que un $76,7 \%$ de los pacientes tenía algún grado de limitación. Esto puede deberse no sólo a la insuficiencia renal, sino también a las complicaciones que conlleva esta patología. Por lo tanto, la actividad física que pue- den realizar estos pacientes siempre debe ser aconsejada en forma individual, tomando en cuenta factores como su situación clínica, edad, tipo detratamiento depurador y las pre ferencias. El rol físico alcanzó una de las medias más bajas dela población en estudio, 23,6 (DT:34), y se pudo observar que más de un $70 \%$ de los pacientes declaró que su salud física interfiere en el desempeño de su trabajo, lo que coincide con los resultados de otros estudios si milares y publicados en revistas de circulación internacional, esto es, que cifras cercanas a las dos terceras partes de los pacientes en diálisis no vuelven al trabajo que realizaban antes del inicio de la insuficiencia renal crónica terminal.

El dolor que acusa el $64,4 \%$ se presenta durante la sesión de hemodiálisis y con posterioridad a ella. Durante la sesión se presenta como contracturas o calambres musculares, específicamente en extremidadesinferio- 
res, superiores y dorso, seguidos por dolor abdominal y cefalea; posterior a ella los dolores óseos, musculares, el síndrome del túnel carpiano, cefalea y dolor abdominal son los más referidos por los pacientes.

En relación a la percepción de la salud general informada por estos pacientes, se aprecia que la autovaloración realizada de su situación personal respecto a las perspectivas de su salud en el futuro y la resistencia a enfermar, es regular a baja, lo que es comprensible por la experiencia que están enfrentando actualmente.

La vitalidad y energía presentó una de las medias más bajas de este estudio, lo que se relaciona con las complicaciones crónicas que sufre este tipo de paciente, como son los trastornos cardiovasculares presentados por el $80 \%$, anemia en un $90 \%$ de los casos y trastornos de la nutrición y del metabolismo.

En relación a la función social, se pudo observar quela gran mayoría de los pacientes no había cambiado sus redes sociales al surgir la enfermedad. De acuerdo a lo conversado con ellos, se puede destacar una marcada diferencia entre los que viven en sectores rurales, quienes se sienten mucho mas integrados a las actividades cotidianas, que los residentes en sectores urbanos, quienes perciben una cierta discriminación por parte de la comunidad.

Como se ha observado en este estudio, la familia, considerada como red de apoyo social, constituyeel pilar fundamental deapoyo al paciente con enfermedad crónica, afirmación sostenida por el $100 \%$ de los pacientes que tenían cobertura familiar.

Esto confirmaría que en la familia los pacientes se sienten protegidos y acompañados, con lo cual sus miedos se disipan y su angustia disminuiría en función de la respuesta positiva del apoyo social de su familia. Si esto no fuese así, el paciente, además de sufrir la enfermedad que físicamente le disminuye, podría ir espiritualmenteperdiendo sus facultades esenciales (de pertenencia, apoyo, ener- gía, vitalidad, etc.) y alejándose cada vez más de su personalidad anterior.

\section{REFERENCIAS BIBLIOGRÁFICAS}

Alonso J. y L. Prieto. (1995). Versión española del SF-36, Cuestionario de Salud, un instrumento para la medidade resultados clínicos. Med. Clin. Barcelona. 104:771-776 págs. Web site: http:// www.imim.es

Alvarez, U. de F.; Vicente, E. y Badía X. (1995). La medida de la calidad de vida relacionada con la salud en los pacientes en programa dehemodiálisisy diálisisperitoneal continua ambulatoria de Segovia. Revista de N efrología, Vol 15, № 6. 572$580 \mathrm{pp}$.

Bravo, M. y Falache, S. (1993). Un concepto deCalidad deVida. Una revisión de su al cancey contenido. Revista Geográfica Venezolana, Vol. 34. 275$295 \mathrm{pp}$.

Croog, S. y Levine, S. (1989). Quality of Life and $\mathrm{H}$ ealth Care Interventions $\mathrm{H}$. Freeman y Sol Levine (eds.). Handbook of Medical Sociology ( $2^{\mathrm{a}}$ ed.) N ew Jersey Prentice Hall (236-261 pp.). Gómez, M . y Sabeh, E. (2000). Calidad devida. Evolución del concepto y su influencia en la investigación y la práctica. Instituto Universitario delntegración en la Comunidad, Facultad de Psicología, Universidad de Salamanca. M adrid, Rialp.

Iborra, M . Carmel o y Corbí, R. Daniel. (1998). Calidad de vida de los pacientes dializados: Revisión bibliográfica. Revista de la Sociedad Española de Enfermería N efrológica. Cuarto trimestre, $\mathrm{N} \cong 4$, $58 \mathrm{pp}$.

INE, Instituto Nacional de Estadística (2001). Boletín de empleo $\mathrm{N}^{\circ}$ 8, edición $\mathrm{N}$ 욱. trimestreenero-marzo.

Jiménez, S. y M . I gnacia. (1998). Intentando definir la Calidad deVida. Revista ETS Trabajo Social , Pontificia Universidad Católica de Chile № 54, 24-31 pp.

Lavados, J. (1983). D esarrollo biológico y calidad de vida. RevistaC.P.U. (Corporación dePromoción Universitaria) Vol. 35. 65-83 pp.

Lugones, M . (2002). Algunas consideraciones sobre la calidad de vida. Revista Cubana M edicina General Integral, 4.

Moreno F.( 1996). Quality of life in Diálisis Patients. A Spanish, Multicentre Study. Nephhrol Dial Transplant, 11 Suppl 2: 125-129 pp.

Poblete, B. (1999). Hemodiálisis crónica en Chile. Cuenta deH emodiálisis Crónica en Chile. 51 pp. 
Palomino, B. y López, G. (1999). Reflexiones sobre la calidad de vida y el desarrollo. Región y Sociedad. 11 (17).

Rebollo, P.; Bobes, J.; González M .; Saiz, P. y O rtega, F. (2000). Factores asociados a la Calidad deVida Relacionada con la Salud, de los pacientes en terapia renal sustitutiva(TRS) v. Departamento de M edicina. Área de Psiquiatría de la Universidad de Oviedo. Servicio de N efrología-I del Hospital Central. Revista de N efrología. Originales. Volumen $X X, N$ 으. 2 .

Schwartzmann, L. y Olaizola, I. (1999). Validación de un instrumento para medir calidad de vida en hemodiálisiscrónica. Revista M édica Uruguay, Vol. 15. №2. E-mail:lauras@chasque.apc.org

Schwartzmann, L. (2003). Calidad de vida relacionada con la salud: aspectos conceptuales. Ciencia y Enfermería. 2.www.udec.cl/publicaciones/cye

Valderrábano, Fernando (1999). Tratado de hemodiálisis. Editorial Medical Jims S.L. $1^{\text {a }}$ edición. Barcelona, España.

Viñas, Ángel (1981). M edio ambiente y calidad de vida. Documentación Administrativa, abril-junio, 7-36 pp.

Velarde, E. Ávila, C. (2002). Evaluación dela calidad de vida. Salud Pública de M éxico, 44 (4), 349361.

Ware, J.E. y Sherbourne, C. (1993). SF-36 Health Survey. M anual and Interpretation Guide. Boston M .A:TheH ealth Institute, N ew England M edical Center.

Zanoguera, G. M ercedes (1998). Calidad de vida en pacientes en hemodiálisis. Trabajo presentado a la Revista del XXIII Congreso Nacional de la Sociedad Española de Enfermería N efrológica, Editada. Hospal S.A Nápoles, 249 Barcelona. Sevilla. $480 \mathrm{pp}$.

Ureña, A.; Rovira, P.; Ramos, J. y Espí, M . (1997). Evolución de la calidad de vida relacionada con la salud en las personas portadoras de un riñón trasplantado. Trabajo presentado a la Revista del XXII Congreso Nacional dela Sociedad Española de Enfermería N efrológica. Editorial Hospal, S.A., Nápoles, 249. Barcelona. Santander, 5-8 de octubre. 